\title{
ABC of Blood Pressure Measurement
}

EOIN T O'BRIEN KEVIN O'MALLEY

\section{THE SPHYGMOMANOMETER}

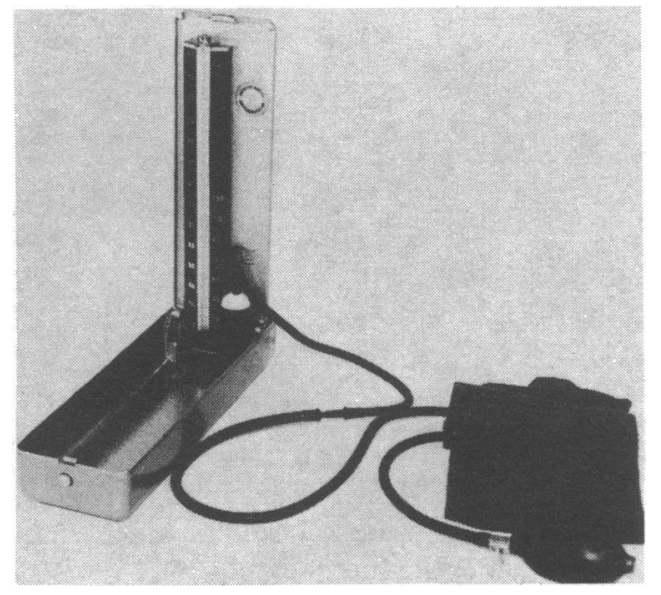

The sphygmomanometer is an indispensable piece of medical diagnostic equipment, but all too often its continuing efficiency is taken for granted. A survey of all our hospital sphygmomanometers showed that nearly half of them were inaccurate, and similar results have been found in other hospitals. The proportion is probably little different in general practice. Few hospitals have any policy for the regular maintenance of basic equipment such as sphygmomanometers.

\section{Mercury manometers}

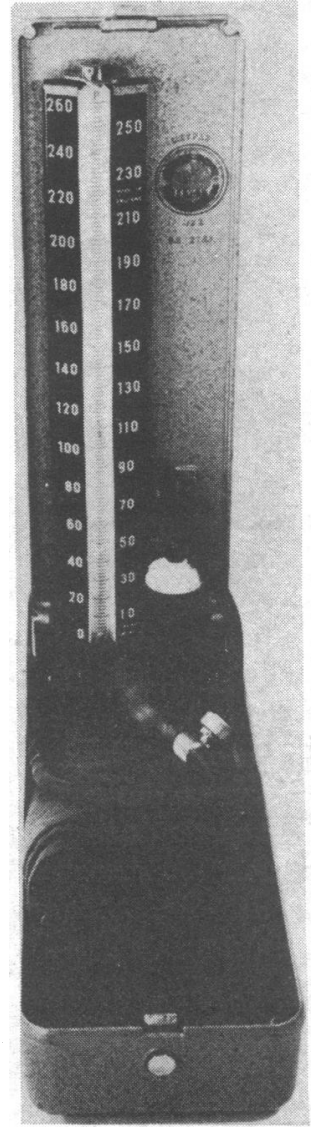

Mercury sphygmomanometers are reliable pieces of equipment that are easily maintained. Assessed against direct intra-arterial pressures, the standard mercury-in-glass manometer tends to underestimate systolic pressure by about $10 \mathrm{~mm} \mathrm{Hg}$ and to overestimate diastolic (phase 5) pressure by about $8 \mathrm{~mm} \mathrm{Hg}$.

The top of the mercury meniscus should rest at exactly zero without pressure applied; if it is below this mercury needs to be added to the reservoir. The scale should be clearly calibrated in 2-mm divisions from 0 to $300 \mathrm{~mm} \mathrm{Hg}$ (we hope kilopascals will not be introduced) and should indicate accurately the differences between the levels of mercury in the tube and in the reservoir. The diameter of the reservoir must be at least 10 times that of the vertical tube, or the vertical scale must correct for the drop in the mercury level in the reservoir as the column rises.

Substantial errors may occur if the manometer is not kept vertical during measurement. (Calibrations on floor models are especially adjusted to compensate for the tilt in the face of the gauge.)

The air vent at the top of the manometer must be kept patent since clogging will cause the mercury column to respond sluggishly to pressure changes. Mercury sphygmomanometers need cleaning and checking every year. 


\section{Aneroid manometers}

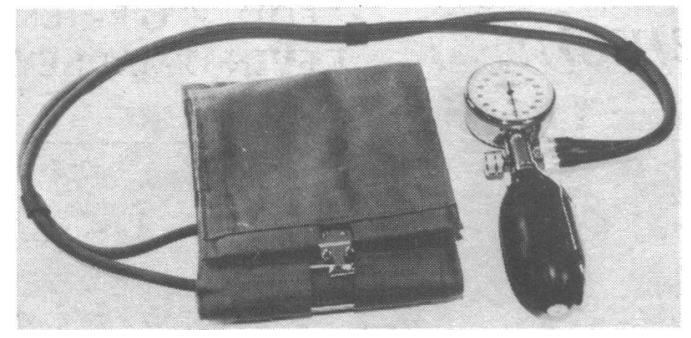

\section{Which manometer?}

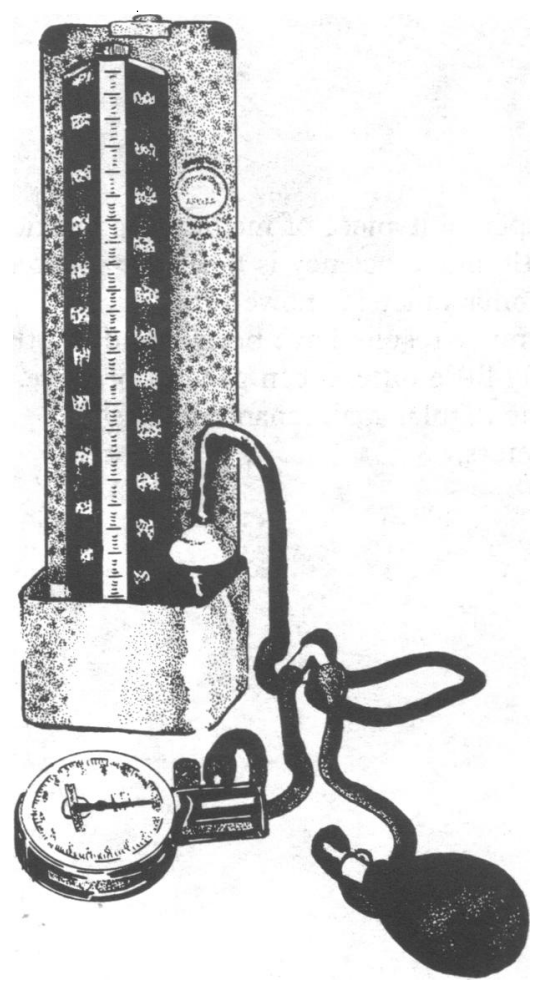

\section{The cuff}

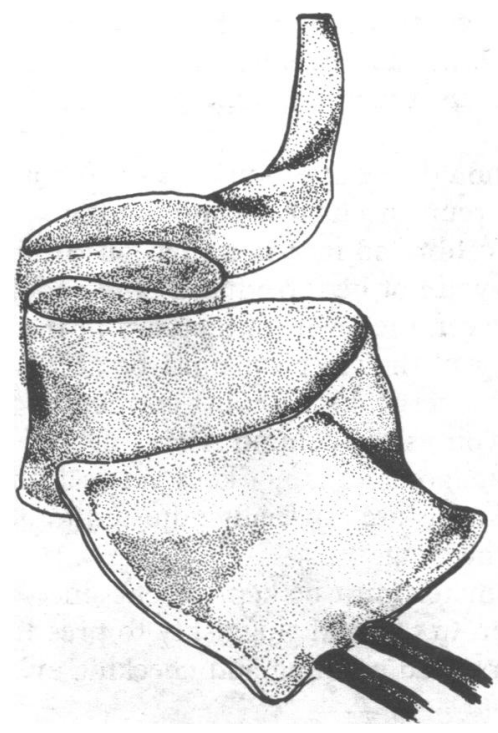

Aneroid sphygmomanometers have levers which can stick and are affected by jolts, and they are generally less accurate than mercury manometers. When calibrated against a mercury manometer an average deviation of $3 \mathrm{~mm} \mathrm{Hg}$ is considered acceptable. Even so, some studies have shown that $30-35 \%$ of aneroid sphygmomanometers have an average deviation of over $3 \mathrm{~mm} \mathrm{Hg}$, while $6-13 \%$ deviate by $7 \mathrm{~mm} \mathrm{Hg}$ or more.

Some new aneroid sphygmomanometers incorporate a gauge which indicates when the machine is losing accuracy.

The choice of sphygmomanometer should be influenced by the use to which it will be put, the care with which it will be treated, and the availability of facilities for regular maintenance. When a machine will be used by many observers and is therefore likely to be knocked or jolted, and when maintenance facilities are not available, the mercury sphygmomanometer is better than the aneroid machine. Aneroid instruments should be checked against a mercury manometer every six months, and the aneroid manometer needs to be checked throughout the entire pressure range.

The cuff is an inelastic cloth that encircles the arm and encloses the inflatable rubber bladder. It is secured round the arm by wrapping its tapering end into the encircling cuff, by Velcro surfaces, or by hooks.

Tapering cuffs should be long enough to encircle the arm several times: the full length should extend beyond the end of the inflatable bladder for $25 \mathrm{~cm}$ and then should gradually taper for a further $60 \mathrm{~cm}$. Velcro surfaces must be effective, and when they lose their grip the cuff should be discarded. 


\section{The inflatable bladder}

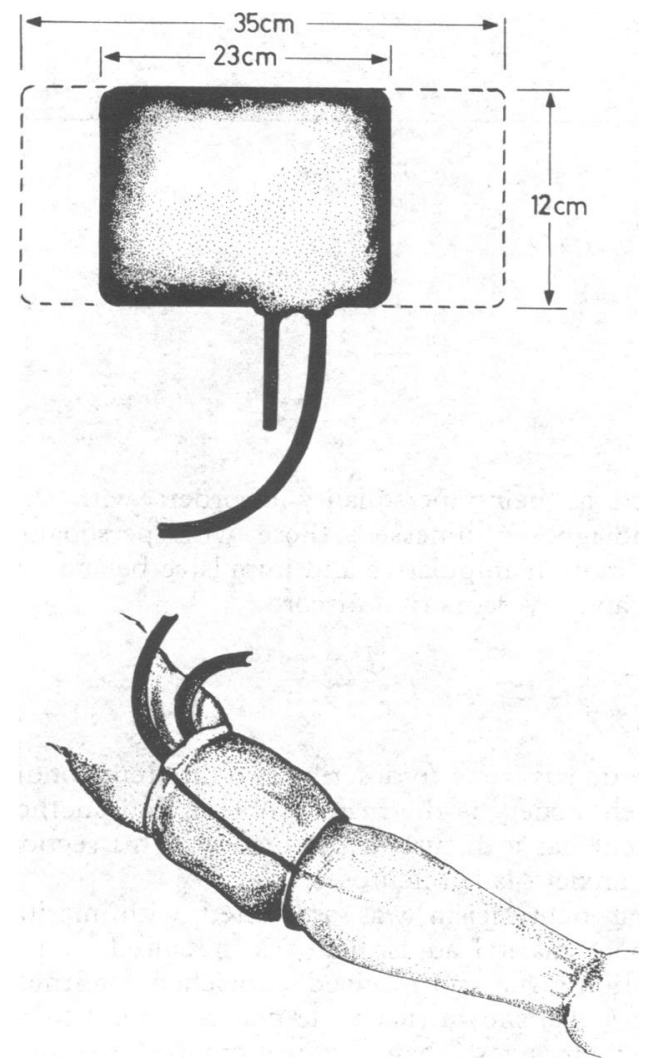

A bladder which is too short or too narrow, or both (overall too small), will give falsely high pressures, and one which is too wide or too long, or both (overall too large), will give falsely low pressures. Nevertheless, if the bladder is too wide or too narrow only a small loss of accuracy results so long as the bladder is long enough to encircle the arm completely. Readings taken with a completely encircling bladder correlate best with intra-arterial pressure.

The recommended bladder width is $20 \%$ greater than (or 1.2 times) the diameter of the limb, which is equivalent to $40 \%$ of the arm's circumference. Most bladders are 12 to $13 \mathrm{~cm}$ wide, with those for obese arms being 15 or $16 \mathrm{~cm}$, but most arms will not comfortably accommodate a cuff much wider than $13 \mathrm{~cm}$.

Bladder iengths vary from around 22 to $36 \mathrm{~cm}$, there being different lengths for obese arms, for children's arms, and for thighs. The standard bladder $(22.9 \mathrm{~cm}$ long) will encircle only $33 \%$ of adult arms, whereas a $36 \mathrm{~cm}$ bladder would encircle $99 \%$ of adult arms.

Manufacturers of blood pressure equipment must be persuaded to review bladder size. We recommend that for adults the bladder should be at least $35 \mathrm{~cm}$ long and $12 \mathrm{~cm}$ wide-a size which is not readily available.

If the bladder does not encircle the arm, the centre of the bladder must be placed directly over the artery to be compressed, or the readings will be very inaccurate. Many cuffs now indicate the centre of the bladder and also indicate bladder size in relation to arm circumference.

\section{Rubber tubing, pump, and control valve}

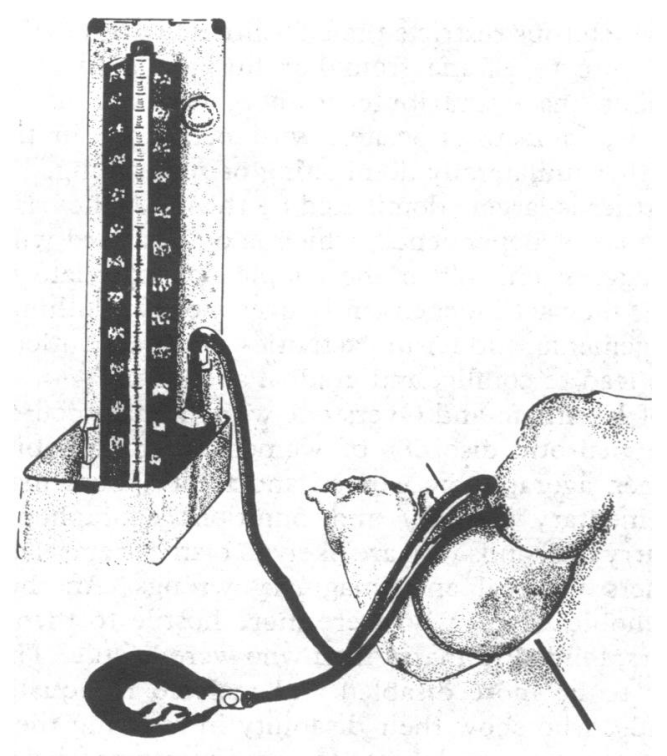

Leaks due to cracked or perished rubber make accurate measurement of the blood pressure difficult because the fall in mercury cannot be controlled. The rubber should be in a good condition and free of leaks. The tubing should be at least $76 \mathrm{~cm}$ long, and connections must be airtight and easily disconnected.

The control valve is a common source of error, especially in sphygmomanometers with an air filter, rather than a rubber valve. The filter may become blocked with dirt, which demands excessive squeeze on the pump. The control valve should allow the passage of air without excessive effort; when closed it should hold the mercury at a constant level; and when released it should allow a controlled fall in the level of mercury.

The valve may be tested by rolling a cloth cuff into its own tail or, with a Velcro cuff, matching Velcro to Velcro, and then pumping up to $200 \mathrm{~mm} \mathrm{Hg}$ and waiting for 10 seconds. The mercury should not fall more than $2 \mathrm{~mm} \mathrm{Hg}$ during those 10 seconds. If it does fall further the circuit should be clamped in sections to locate the leak, which is usually in the control valve. The valve should then be released slowly four times, on two

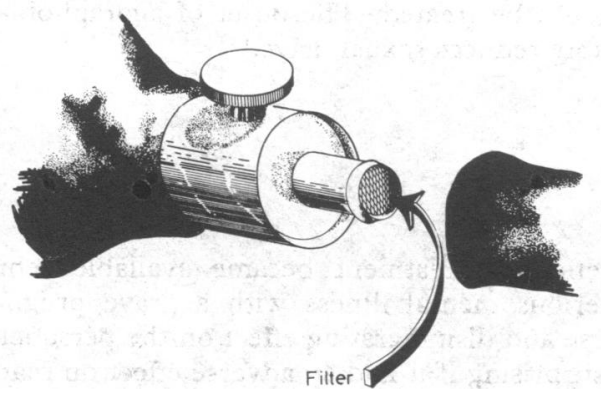
of which it should be possible to control the rate of fall to $1 \mathrm{~mm} \mathrm{Hg}$ per second, and to change from faster to slower rates at will. Inability to do so is usually due to air filter blockage, which may be rectified by cleaning the filter.

The Blood Pressure Evaluation and Treatment Centre, The Charitable Infirmary, Dublin: Dr Eoin O'Brien is consultant physician (cardiology) and Professor Kevin O'Malley professor of clinical pharmacology at the Royal College of Surgeons in Ireland.

This is the second of a series of seven papers, and no reprints will be available from the authors. 